\title{
Spectral branes
}

\author{
D.V. Vassilevich* \\ University of Leipzig, Institute for Theoretical Physics \\ Augustusplatz 10/11, 04109 Leipzig, Germany
}

October 25, 2018

\begin{abstract}
We study the objects (called spectral branes or $S$-branes) which are obtained by imposing non-local spectral boundary conditions at the boundary of the world sheet of the bosonic string. They possess many nice properties which make them an ideal test ground for the string theory methods. Depending on a particular choice of the boundary operator $S$-branes may be commutative or non-commutative. We demonstrate that projection of the $B$-field on the brane directions (i.e. on the components which actually influence the boundary conditions) is done with the help of the chirality operator. We show that the $T$-duality transformation maps an $S$-brane to another $S$-brane. At the expense of introducing non-local interactions in the bulk we construct also a duality transformation between $S$-branes and $D$-branes or open strings.
\end{abstract}

\section{Introduction}

Open strings with Dirichlet conditions at the boundary of the world sheet [1, 2] were first considered as a rather exotic object. However, a few year later [3] these objects (the $D$-branes) became a center of a very important development in the string theory. Therefore, it seems natural to attempt to introduce different boundary conditions in string theory and to study some properties of the resulting objects.

In the present paper we suggest to impose a non-local spectral boundary conditions on coordinates of the endpoints of open bosonic string. Such boundary conditions we introduced by Atiyah, Patodi and Singer [4] to study the Index

*On leave from V.A. Fock Department of Theoretical Physics, St. Petersburg University, 198904 St. Petersburg, Russia. e-mail: Dmitri.Vassilevich@itp.uni-leipzig.de 
Theorem. Since that time they appeared in various mathematical and physical contexts (see e.g. [5 9] and references therein). Strictly speaking, spectral boundary conditions were suggested for fermion theories. Here we extend the scheme a little bit to use it for the bosonic strings. To this end, we represent the Laplace operator which appears in the equations of motion through the Dirac operators. In the simplest case this may be done explicitly. For more complicated background field we argue that such representation is possible.

We call the object which is obtained by imposing spectral boundary conditions on the boundary of the world sheet the spectral brane (or $S$-brane, for short). It looks like a non-local mixture of the $D$-brane and the open strings states. Namely, if we define a "boundary helicity operator" (see eq. (8), the positive helicity states are taken from $D$-brane, while the negative helicity ones - from the open string. The $S$-brane shares some nice properties with more common string models. For example, the string coordinates may be non-commutative for a suitable choice of the boundary operator. As for the $D$-brane, only part of the components of the $B$-field enters actually the boundary conditions. Projection on the "brane directions" is local (even though the boundary conditions are nonlocal) and is done with the help of a chirality matrix $\gamma_{5}$ which appears naturally in the construction. Different parity properties of the $\gamma_{5}$ suggest that the low-energy effective theory may differ substantially from that for $D$-branes.

Duality transformations play a central role in string theory. They relate strong and weak coupling limits of various string models. It is known that the $T$-duality interchanges Dirichlet and Neumann boundary conditions. Since the $S$-brane is a non-local mixture of the Dirichlet and Neumann states, under $T$-duality it is transformed to another $S$-brane. This means that $S$-branes may occupy a special place among other string/brane models. It is also interesting that by moving nonlocality to the bulk action we may even define duality transformation between $S$-branes and $D$-branes or open strings.

The paper is organised as follows. In next section we consider some consistency requrements which should be satisfied by the boundary conditions and formulate spectral boundary conditions for the bosonic string. We also find a local projector of the $B$-field on $S$-brane and discuss non-commutativity of the string coordinates. Sec. 3 is devoted to $T$-duality. We construct in a standard way the duality transformation which maps $S$-branes to $S$-branes and comment on $S$-brane - $D$-brane and $S$-brane - open string duality. Sec. 4 contains discussion of the results and perspectives. Some mathematical statements on the relations between Dirac and Laplace operators are collected in the Appendix.

Throughout this paper we consider the simplest possible geometry of the string. We suppose that the target space metric is constant. In sec. 2 we also add a constant $B$-field. 


\section{Boundary conditions}

Consider the action for the open bosonic string:

$$
S=\frac{1}{2} \int_{M} d^{2} x \sqrt{h} h^{a b} G_{\mu \nu} \partial_{a} X^{\mu} \partial_{b} X^{\nu}
$$

The string tension $\alpha^{\prime}$ has been absorbed in $G_{\mu \nu}$. For simplicity, we suppose that the target space metric $G_{\mu \nu}$ is constant and the world-sheet metric $h$ is flat. We also assume Euclidean signature on the world sheet and on the target space. Variation of the action (1) with respect to $X$ gives:

$$
\delta S=\int_{M} d^{2} x \sqrt{h} G_{\mu \nu}\left(\delta X^{\mu}\right)\left(-\nabla_{a} \nabla^{a}\right) X^{\nu}-\int_{\partial M} d \tau\left(\delta X^{\mu}\right) \partial_{\sigma} X^{\nu} G_{\mu \nu}
$$

where $\tau$ is the proper distance along the boundary. $\partial_{\sigma}$ denotes partial derivative with respect to an inward pointing unit normal. The first (volume) term in (2) generates the equations of motion, while the second term should vanish due to the boundary conditions.

There are two consistent choices of local boundary conditions. These are Dirichlet $\left(\left.\delta X\right|_{\partial M}=0\right)$ or Neumann $\left(\left.\partial_{\sigma} X\right|_{\partial M}=0\right)$ conditions. One can also impose different conditions on different components of $X^{\mu}$ thus constructing various D-branes.

It we allow the boundary conditions to be non-local our choice becomes much wider. On and near the boundary one can decompose $X$ into a sum of orthogonal functions

$$
X^{\mu}(\sigma, \tau)=\sum_{n} \chi_{n}^{\mu}(\tau) c_{n}(\sigma), \int_{\partial M} d \tau \chi_{n}^{\mu} \chi_{m}^{\nu} G_{\mu \nu}=\delta_{n m},
$$

so that the surface integral in (2) becomes a sum over $n$ of a product of the boundary values of the Fourier coefficients $c_{n}$ and their normal derivatives:

$$
\sum_{n}\left(\delta c_{n}(0)\right) \partial_{\sigma} c_{n}(0)
$$

$\sigma=0$ is the boundary. To cancel this boundary term one should require that for any $n$ either $\delta c_{n}$ or $\partial_{\sigma} c_{n}$ vanishes at the boundary. This means that one may impose Dirichlet or Neumann boundary conditions on different harmonics independently.

In this way one can construct an infinite variety of boundary conditions. Most of them are too exotic to be physically relevant. We restrict ourselves to the spectral boundary conditions introduced by Atiyah, Patodi and Singer [4] in their study of the index theorem for manifolds with boundary. These boundary conditions are defined by an operator of Dirac type associated with the Laplacian 
on $M$. In order to define this operator let us introduce a moving frame $E_{\mu}^{A}$ on the target space and let $X^{A}=E_{\mu}^{A} X^{\mu}$. The equations of motion now become

$$
\Delta X^{A}=0, \quad \Delta:=-\nabla^{a} \nabla_{a} .
$$

Suppose that the target space is even dimensional. We introduce $\gamma$-matrices such that

$$
\left(\gamma_{a} \gamma_{b}+\gamma_{b} \gamma_{a}\right)^{A B}=-\delta^{A B} h_{a b} .
$$

For more than two target space dimensions any representation of the Clifford algebra (6) is reducible. Effectively, one should decompose the target space into a direct sum of two-dimensional subspaces and define standard $\gamma$-matrices on each subspace. Then

$$
\Delta=P^{2}, \quad P=\gamma^{a} \nabla_{a} .
$$

Let us define a first order differential operator on the boundary $\partial M$

$$
\mathcal{B}:=\gamma_{\sigma} \gamma^{\tau} \nabla_{\tau}
$$

which we call boundary helicity operator. This operator is self-adjoint and, therefore, has a real spectrum. Let $\Pi_{+}, \Pi_{0}$ and $\Pi_{-}$denote projectors on the spaces with positive, zero and negative eigenvalues of $\mathcal{B}$ respectively. Spectral boundary conditions are defined as:

$$
\left.\delta(\Pi X)\right|_{\partial M}=0,\left.\quad \partial_{\sigma}(1-\Pi) X\right|_{\partial M}=0 .
$$

We will consider two cases: $\Pi=\Pi_{+}$and $\Pi=\Pi_{+}+\Pi_{0}$.

There is an inhomogeneous term in the Dirichlet part of the boundary conditions (9) $\left.\Pi\left(X-X^{(0)}\right)\right|_{\partial M}=0\left(X^{(0)}\right.$ is fixed, $\left.\delta X^{(0)}=0\right)$. Due to the presence of this term solutions of (9) do not form a linear space. To discuss hermiticity and Green functions we should replace this problem by an associated linear problem. This can be done by using the background field formalism (see below). In the present context we can simply use translational invariance to put $X^{(0)}=0$. We arrive at linear homogeneous boundary conditions:

$$
\left.(\Pi X)\right|_{\partial M}=0,\left.\quad \partial_{\sigma}(1-\Pi) X\right|_{\partial M}=0 .
$$

Note, that with the conditions (10) $\Delta$ is formally self-adjoint.]

Requiring that coordinates of the endpoints of a string satisfy the conditions (10) we obtain a new object which we call spectral brane. It is constructed from positive helicity states of the $D$-brane system and negative helicity states of the

\footnotetext{
${ }^{1}$ In spectral theory of the Dirac operator instead of the second boundary condition in (10) it is common to require $\left.\Pi P X\right|_{\partial M}=0$. This guarantees existence of the spectrum of $P$. In our construction the Dirac operator plays an auxiliary role. It is more important that the variation problem (2) is well defined. For this reason we have to choose the second boundary condition as it is written in (10).
} 
open string. The boundary conditions (10) are non-local. This suggests that we are dealing with certain collective excitations of fundamental strings.

Let us switch on the $B$ field by adding to the action (11) the following term

$$
S_{B}=\frac{i}{2} \int_{M} d^{2} x \epsilon^{a b} B_{\mu \nu} \partial_{a} X^{\mu} \partial_{b} X^{\nu}
$$

Again, we suppose for simplicity that the $B$ field is constant. Then (11) contributes to the boundary term only in variation of the string action. Total boundary term in this variation now reads:

$$
-\int_{\partial M} d \tau\left(\delta X^{\mu}\right)\left(G_{\mu \nu} \partial_{\sigma}+i B_{\mu \nu} \partial_{\tau}\right) X^{\nu}
$$

Since the volume term in the variation is not changed, there is no need to modify the equation (7) and (8). The boundary conditions which ensure vanishing of the boundary variation (12) now read (after passing to an associated linear problem - see a remark between eqs. (9) and (10) above):

$$
\left.(\Pi X)\right|_{\partial M}=0,\left.\quad(1-\Pi)\left(\partial_{\sigma}+i B \partial_{\tau}\right)(1-\Pi) X\right|_{\partial M}=0 .
$$

Note, that due to the presence of the imaginary unit in front of $B$ in the boundary conditions (13) neither $P$ nor $\Delta$ is self-adjoint. This happens even if the projector $\Pi$ is local as for the $D$-branes. There are two ways to overcome this difficulty. One can either change rules of continuation to the Euclidean space for the $B$ field [11], or assume more complicated conjugation rules in the Euclidean space [12. We take the second option here to be close to commonly accepted notations.

It is interesting to note that only a part of the components of $B$ enter the boundary conditions (13). Let us introduce the $\gamma_{5}$ matrix as $\gamma_{5}=\gamma_{\sigma} \gamma^{\tau}, \gamma_{5}^{2}=-1$. We split $B$ into odd end even parity parts with respect to this $\gamma_{5}$ :

$$
B=B^{(+)}+B^{(-)}, \quad B^{( \pm)}=\frac{1}{2}\left(B \pm \gamma_{5} B \gamma_{5}\right), \quad \gamma_{5} B^{( \pm)}=\mp B \gamma_{5}
$$

$B^{(-)}$commutes with $\Pi_{-}, \Pi_{+}$and $\Pi_{0} . B^{(+)}$intertwines $\Pi_{+}$and $\Pi_{-}: B^{(+)} \Pi_{+}=$ $\Pi_{-} B^{(+)}$. We see, that in the boundary conditions $(13)$ the components $B^{(+)}$can interact with $\Pi_{0} X$ only (if $\Pi_{0}$ is included in $1-\Pi$ ), i.e. with zero modes of $\partial_{\tau}$. However, if $\left.\partial_{\tau} X\right|_{\partial M}=0$ the term with $B$ in the boundary conditions (13) vanishes. We conclude, that $B^{(+)}$drops out completely. $B^{(-)}$plays a role of a projection of $B$ on the brane directions. An unexpected feature is that this projection is local in the target space, as in the $D$-brane case, even though the boundary conditions are non-local. In the rest of the paper we suppose $B^{(+)}=0$ and, therefore, $B=B^{(-)}$.

To discuss the non-commutativity issues we need a convenient representation for the propagator. Let us put temporarily our system in a box in the $\tau$-direction 
so that spectrum of $\partial_{\tau}$ becomes discrete. Then

$$
\begin{aligned}
\Pi_{+} & =\frac{1}{2 \pi i} \lim _{\mu \rightarrow+0} \int_{-\infty}^{\infty} \frac{d \alpha}{\alpha-i \epsilon} \exp (i \alpha(\mathcal{B}+\mu)) \\
\Pi_{+}+\Pi_{0} & =\frac{1}{2 \pi i} \lim _{\mu \rightarrow+0} \int_{-\infty}^{\infty} \frac{d \alpha}{\alpha-i \epsilon} \exp (i \alpha(\mathcal{B}-\mu))
\end{aligned}
$$

with the boundary helicity operator $\mathcal{B}(8)$. $\Pi_{-}$can be constructed in the same way. Now we take the limit of an infinite size of the box and consider (15) as definitions of the projectors for that case.

We can extend the projectors $\Pi$ and $(1-\Pi)$ inside the manifold $M$ to a neighbourhood of $\partial M$. For $x$ and $y$ in this neighbourhood, the propagator with the boundary conditions (13) is

$$
\langle X(x) X(y)\rangle=\Pi_{x} \mathcal{G}_{D}(x, y) \Pi_{y}+(1-\Pi)_{x} \mathcal{G}_{N}(x, y)(1-\Pi)_{y},
$$

where $\mathcal{G}_{D}$ and $\mathcal{G}_{N}$ are the standard propagators with Dirichlet and generalized Neumann boundary conditions:

$$
\left.\mathcal{G}_{D}(x, y)\right|_{x \in \partial M}=0,\left.\quad\left(\partial_{\sigma}^{(x)}+i B \partial_{\tau}^{(x)}\right) \mathcal{G}_{D}(x, y)\right|_{x \in \partial M}=0
$$

If $x$ and $y$ are on the boundary, only the Neumann part of the propagator [13, 14,

$$
\mathcal{G}_{N}\left(\tau, \tau^{\prime}\right)=-D \log \left(\tau-\tau^{\prime}\right)^{2}+\frac{i}{2} \theta \epsilon\left(\tau-\tau^{\prime}\right)
$$

survives. Here $\epsilon$ is the sign function and

$$
D=\left(1-B^{2}\right)^{-1}, \quad \theta=-B\left(1-B^{2}\right)^{-1}
$$

are symmetric and antisymmetric matrices respectively. It can be demonstrated that the $D$-part of the propagator remains symmetric in $\tau$ and $\tau^{\prime}$ after the action of the projectors $(1-\Pi)$. This part does not contribute to the time-ordered product. Let $\tau \neq \tau^{\prime}$. Then $\mathcal{B}_{\tau} \theta \epsilon\left(\tau-\tau^{\prime}\right)=0$. Hence only the $\mu$-term remains in the exponential in the integral representation for the projectors (15). This means

$$
(1-\Pi)_{\tau} \theta \epsilon\left(\tau-\tau^{\prime}\right)(1-\Pi)_{\tau^{\prime}}=\theta \epsilon\left(\tau-\tau^{\prime}\right)
$$

if projector on the zero modes $\Pi_{0}$ is included in $(1-\Pi)$, and

$$
(1-\Pi)_{\tau} \theta \epsilon\left(\tau-\tau^{\prime}\right)(1-\Pi)_{\tau^{\prime}}=0
$$

otherwise. Consequently, we reproduce the famous relations for a non-commutative space 13, 14

$$
\left[X(\tau), X\left(\tau^{\prime}\right)\right]=i \theta
$$


in the former case $\left((1-\Pi) \Pi_{0}=\Pi_{0}\right)$ and loose the non-commutative structure in the latter one $\left((1-\Pi) \Pi_{0}=0\right)$.

In a non-linear theory (as if $G_{\mu \nu}$ and $B_{\mu \nu}$ are not constants) one can use the background field formalism and repeat this construction with the part of the action which is quadratic in quantum fluctuations. Let us outline briefly how the above procedure can be generalized. First we split $X=\bar{X}+\xi$ into a background part $\bar{X}$ and fluctuations $\xi$. The propagator is defined by the quadratic part of the action:

$$
S_{2}=\int_{M} d^{2} x \sqrt{h} \xi D \xi+\int_{\partial M} d \tau \xi\left(-\partial_{\sigma}+L\right) \xi,
$$

where $D$ and $L$ are some operators. Next we represent $D$ as

$$
D=P^{\dagger} P+c
$$

with a Dirac operator $P$ and a constant $c$ and construct the projector $\Pi$ on the Dirichlet components for the corresponding elliptic complex of Dirac type (see [1] for the details). Boundary conditions read:

$$
\left.\Pi \xi\right|_{\partial M}=0,\left.\quad(1-\Pi)\left(-\partial_{\sigma}+L\right)(1-\Pi) \xi\right|_{\partial M}=0
$$

Some comments are in order. In Appendix, by using a kind of the degrees of freedom counting, we argue that the representation (24) is generically possible at least locally. From that consideration it is clear that explicit construction the operator $P$ for any given $D$ is extremely complicated. This however seems to be not a very serious drawback. First, eq. (24) can be viewed as another parametrisation of the background fields in the closed string sector. Second, constructions of this type become much easier in the presence of supersymmetry of the background. And third, the operator $P$ plays an auxiliary role. It may be not necessary to have an explicit solution of (24) everywhere on $M$.

\section{T-duality}

Action of $T$-duality transformations on open string theories has been studied in [1, 2, 15, 16] and since then became a very popular subject mostly due to the Polchinski's idea [3] that $D$-branes may carry R-R charges. In this section we follow the approach of [17] based on gauging the space-time isometries of the open string (see also related works [18, 19]). Since in our construction two-dimensional blocks play a special role, we need two commuting isometries acting along the directions $X^{\alpha}=k_{\mu}^{\alpha} X^{\mu}, \alpha=1,2 . k_{\alpha}$ are the Killing vectors. We suppose that mixed comonenets of the target space metric are absent, $G_{\alpha \mu}=0, \mu \neq 1,2$. We choose representation of the Clifford algebra in such a way that $X^{1}$ and $X^{2}$ belong to one irreducible piece. With this choice, boundary conditions for $X^{\alpha}$ become fully decoupled from that for the other components and can be considered 
separately. Note, that the boundary conditions for $X^{\mu}, \mu \neq 1,2$ should not be necessarily spectral. We can consider a configuration which look as an open string or as a $D$-brane from that directions.

For simplicity we neglect the $B$-field. The action for open strings with gauged isometries reads:

$$
S_{\text {gauged }}=\frac{1}{2} \int_{M} d^{2} x\left(G_{\mu \nu} D^{a} X^{\mu} D_{a} X^{\nu}+i Y_{\alpha} \epsilon^{a b}\left(\partial_{a} V_{b}^{\alpha}-\partial_{b} V_{a}^{\alpha}\right)\right),
$$

where $D_{a} X^{\mu}=\partial_{a} X^{\mu}+k_{\alpha}^{\mu} V_{a}^{\alpha}$. The action (26) possesses a local symmetry

$$
\delta X^{\mu}=\varepsilon^{\alpha} k_{\alpha}^{\mu}, \quad \delta V_{a}^{\alpha}=-\partial_{a} \varepsilon^{\alpha} .
$$

The auxiliary field $Y^{\alpha}$ generates the constraint

$$
\left(\partial_{a} V_{b}^{\alpha}-\partial_{b} V_{a}^{\alpha}\right) \epsilon^{a b}=0
$$

which eliminates the gauge field $V$ and reduces (26) to the standard action (四). On the other hand, the symmetry (27) allows to gauge away two components $X^{1}$ and $X^{2}$. After integrating by parts the action for $V$ becomes purely algebraic. Eliminating $V$ by means of its' equations of motion

$$
G_{\alpha \beta} V_{b}^{\beta} h^{a b}=i \epsilon^{b a} \partial_{b} Y_{\alpha}
$$

one arrives at a dual action with $Y_{\alpha}$ instead of $X^{1,2}$ :

$$
S_{\text {dual }}=\frac{1}{2} \int_{M} d^{2} x\left(G_{j k} \partial^{a} X^{j} \partial_{a} X^{k}+G^{\alpha \beta} \partial^{a} Y_{\alpha} \partial_{a} Y_{\beta}\right)
$$

where $j, k=3, \ldots, d$. The target space metric is inverted along direction of the first two coordinates.

In the presence of boundaries we should take care of proper boundary conditions for all fields. Let the field $X^{\alpha}$ satisfy Dirichlet boundary conditions. We like to deal with homogeneous boundary value problem, so that $\left.X^{\alpha}\right|_{\partial M}=0$. These boundary conditions are preserved by the gauge transformations (27) if the parameter $\varepsilon$ also vanishes at the boundary, $\left.\varepsilon\right|_{\partial M}=0$. From the transformation low for $V_{a}$ it follows immediately that $\left.V_{\tau}\right|_{\partial M}=0$. A bit more care is needed to define boundary conditions for $V_{\sigma}$. Let us introduce a basis in the functional space of the gauge parameters $\epsilon$ which (similarly to that for the $X$ ) consists of eigenfunctions of the Laplace operator satisfying Dirichlet boundary conditions: $\Delta \epsilon_{\lambda}=\lambda \epsilon_{\lambda}$. Then

$$
\left.\partial_{\sigma}\left(\partial_{\sigma} \epsilon_{\lambda}\right)\right|_{\partial M}=\left.\left(-\lambda-\partial_{\tau}^{2}\right) \epsilon_{\lambda}\right|_{\partial M}=0
$$

From (31) and (27) we conclude that $V_{\sigma}$ satisfies Neumann boundary condition?2 In a similar way we may show that the field strength $\partial_{a} V_{b}-\partial_{b} V_{a}$ satisfies Neumann

\footnotetext{
${ }^{2}$ Such mixed conditions on a vector field when the normal component satisfies Neumann and the tangential one - Dirichlet boundary conditions are called relative boundary conditions in the mathematical literature [10].
} 
boundary conditions as well. Since the field strength is subject to boundary conditions, the unrestricted field $Y_{\alpha}$ contains more components than needed to generate the delta-function $\delta\left(\partial_{a} V_{b}-\partial_{b} V_{a}\right)$. To remove this redundancy one should impose some boundary conditions on $Y_{\alpha}$ as well. From the equations of motion (29) it is clear that these should be Neumann boundary conditions. This choice also allows to integrate by part in the second term in (26). Of course, starting with Neumann boundary conditions for $X^{\alpha}$ one will arrive at Dirichlet boundary condition for $Y_{\alpha}$.

We have reproduced the known fact that $T$-duality interchanges Dirichlet and Neumann boundary conditions. The main advantage of our slightly modified standard derivation is that it can be applied to any eigenmode of the operator $\mathcal{B}$ (8) separately. The reason is that $\mathcal{B}$ commutes with all relevant operators. We conclude that $T$-duality interchanges the two by two blocks in the projectors $(\Pi)_{\alpha \beta}$ and $(1-\Pi)_{\alpha \beta}$ in the boundary conditions (10) and, therefore, maps an $S$-brane to another $S$-brane.

If we turn on the $B$-field by gauging the bulk action (11) the equations of motion (29) will be modified accordingly. Consequentyl, the (dualized) $B$-field will enter Neumann boundary conditions in the usual way. Now, $X^{\alpha}$ or $Y_{\alpha}$ can exhibit a non-commuative structure depending on whether the zero modes are included in corresponding projectors on the Neumann sub-spaces (see discussion in the previous section). Since the duality transformation interchanges Dirichlet and Neumann modes, only the coordinates $X^{\alpha}$ or their duals $Y_{\alpha}$ can be noncommutative (but not $X^{\alpha}$ and $Y_{\alpha}$ simultaneously). This goes in parallel with the ordinary $D$-brane - open string duality.

The last question which will be discussed in this section is a possibility to define duality transformation connecting $S$-branes with $D$-branes or $S$-branes with open strings. To be more specific, let in (10) $\Pi=\Pi_{+}$be a projector on positive boundary helicity states. The projector $\Pi_{+}$can be extended inside the manifold $M$. Then we may write the action (26) where only the positive boundary helicity modes of the isometries are gauged assuming that $Y=Y^{+}=\Pi_{+} Y$ and $V=V_{+}=\Pi_{+} V$ also have positive boundary helicity. Since our $T$-duality arguments may be applied mode-by-mode, this model is equivalent to the original $S$-brane. We may eliminate the modes $X_{+}$and $V_{+}$to obtain the dual action:

$$
S_{\text {dual }}=\frac{1}{2} \int_{M} d^{2} x\left(G_{j k} \partial^{a} X^{j} \partial_{a} X^{k}+G^{\alpha \beta} \partial^{a} Y_{\alpha}^{+} \partial_{a} Y_{\beta}^{+}+G_{\alpha \beta} \partial^{a} X_{-}^{\alpha} \partial_{a} X_{-}^{\beta}\right),
$$

where the part of the action containing the non-positive modes $X_{-}$remains intact. All $\alpha$ components of the string coordinates now satisfy Neumann boundary condition, as in the open string theory. The only difference to the standard case is that non-negative boundary helicity states interact with the target space metric $G_{\alpha \beta}$, while the positive helicity states - with the inverse one $G^{\alpha \beta}$. Therefore, the interaction is non-local. We managed to trade a non-locality in the boundary 
conditions to a non-locality in the bulk action. Construction of the $S$-brane $-D$ brane duality goes the same way. Note, that if the bulk fields are self-dual under the Buscher transformations [20] (which amounts to $G^{\alpha \beta}=G_{\alpha \beta}$ in the present simplified case) the action (32) is local again and coincides with the original one (1). Boundary conditions seem to play no role for such configurations.

\section{Conclusions}

In this paper we have introduced a new object, which we call the $S$-brane, by imposing the spectral boundary conditions on the coordinates of the endpoints of the bosonic string. For the simplest geometry of the target space we were able to study this object quite in detail. This construction is self-consistent in the sense that it leads to a hermitian kinetic operator and, hence, to unitary evolution. Depending on the properties of the non-local boundary projector, the $S$-branes may be commutative or non-commutative. We have found that only a part of the components of the $B$-field enters the boundary conditions. The projection on relevant $B$-field components is done by a local projector containing the chirality matrix $\gamma_{5}$. This indicates that the low energy limit will probably contain chiral boundary fields.

For the target spaces admitting two commuting isometries we constructed a $T$-duality transformation of the $S$-brane. This transformation maps $S$-brane to another $S$-brane of the same dimension. This is a rather unusual property for the open string theory. It suggests that $S$-branes may play a special role in string dualities. We have also considered $S$-brane - $D$-brane and $S$-brane - open strings dualities. By that transformations we were able to shift non-locality from the boundary conditions to the bulk action.

Of course, many question regarding the status of these new objects still remain to be answered. Intrinsic non-locality of the $S$-branes indicates that they are certain composite objects. Therefore, it would be an important development to obtain an $S$-brane as a solitonic solution in another string model. Another interesting topic is inclusion of the supersymmetry. We cannot say much on this at the moment, except that the spectral boundary conditions by their construction should be rather friendly to supersymmetry.

A more straightforward development of the formalism presented in this paper would consist in inclusion of more general background fields. To this end one should refine the arguments on the correspondence between Laplace and Dirac operators (see Appendix). The Dirac parametrisation can be considered as an alternative way to introduce background fields in the closed string sector. Exact relations between the two sets of the background fields are non-local and rather complicated. In the view of our duality arguments this is not an unexpected feature. $S$-branes can be obtained by dualization of $D$-branes or open strings at the expense of introducing non-localities in the bulk action. 
As a part of the program related to general interaction with the background one should calculate the $\beta$-functions for the spectral boundary conditions and reproduce, as a first step, the Born-Infeld action for the (projected) $B$-field. This requires development of the heat kernel technique for these boundary conditions. Note, that the boundary conditions (13) lead to perhaps the most complicated boundary value problem appeared so far in physical applications. They contain both non-localities and tangential derivatives. Therefore, calculation of the heat kernel coefficients is not going to be easy, but is definitely doable. To define the $T$-duality transformations at the presence of the dilaton one will also need to calculate the dilaton shift (see [21]). If the heat kernel is known, this can be done by a rather general method of [22].

To conclude, we note that the $S$-branes seem to be at least a good testing ground for various string ideas and methods.

\section{Acknowledgments}

The author is grateful to M. Bordag, K. Kirsten, W. Kummer, E. M. Santangelo and especially to P. Gilkey for discussions and/or comments. This work has been supported by the DFG project BO 1112/11-1.

\section{Appendix: Dirac and Laplace operators}

In this Appendix we argue that on a two dimensional Riemannian manifold $M$ it is generically possible to represent a Laplace operator via a Dirac operator. We start with some definitions following [6]. Let $\mathcal{V}_{1}$ and $\mathcal{V}_{2}$ be two unitary bundles over $M$ and let $P$ be a first order partial differential operator $P: C^{\infty}\left(\mathcal{V}_{1}\right) \rightarrow C^{\infty}\left(\mathcal{V}_{2}\right)$. Let $P^{\dagger}$ be the formal adjoint of $P$. We say that $P$ defines an elliptic complex of Dirac type if the associated second order operators

$$
D_{1}=P^{\dagger} P, \quad D_{2}=P P^{\dagger}
$$

on $C^{\infty}\left(\mathcal{V}_{1}\right)$ and on $C^{\infty}\left(\mathcal{V}_{2}\right)$ are of Laplace type - i.e. if these operators have scalar leading symbol given by the metric tensor on $M$. If $\mathcal{V}_{1}=\mathcal{V}_{2}$ and $P^{\dagger}=P$, than $P$ is said to be an operator of Dirac type. We impose a somewhat weaker restriction on $P$. We identify locally $\mathcal{V}_{1}$ and $\mathcal{V}_{2}$. In a local basis $P=\gamma^{a} \partial_{a}+r$. We suppose that $\left(\gamma^{a}\right)^{\dagger}=-\gamma^{a}$. Then $\gamma$ define a Clifford module structure.

We see that by definition any Dirac operator (or complex) is associated with a formally self-adjoint operator of Laplace type. Now we wish to invert this construction. For a given a hermitian operator $D$ of Laplace type we like to find an elliptic complex of Dirac type such that

$$
D=P^{\dagger} P+c
$$


where $c$ is a constant. We suppose that $\operatorname{dim} \mathcal{V}$ (the target space dimension) is even so that there exists a (reducible) representation of the Clifford algebra of this dimension. There are some global obstructions to this construction. The simplest one is that $P^{\dagger} P$ is non-negative, while $D$ can have a finite number of negative modes. One can overcome this obstruction by choosing $c$ to be the lowest eigenvalue of $D$. In what follows we will not discuss more global properties of the decomposition (34). Our analysis will be purely local.

It can be shown [10] that any operator of Laplace type can be represented as

$$
E=-\left(\nabla^{a} \nabla_{a}+E\right), \quad \nabla_{a}=\partial_{a}+\omega_{a},
$$

with an appropriate connection one-form $\omega$ and an endomorphism (a matrix valued function) $E$. It has been demonstrated [23] that we may decompose

$$
P=\gamma^{a} \tilde{\nabla}_{a}+\psi
$$

where $\psi$ is a matrix valued function and $\tilde{\nabla}$ is a compatible unitary connection. That means $\tilde{\nabla}_{a} \gamma^{b}=0$ and the connection one-form $\tilde{\omega}_{a}$ is anti-hermitian in a suitable basis. Even though there is some arbitrariness in $\tilde{\omega}$ we suppose that $\tilde{\omega}$ is fixed as soon a representation of the Clifford algebra is fixed. We write

$$
P^{\dagger} P=-\tilde{\nabla}^{a} \tilde{\nabla}_{a}+\frac{1}{4}\left[\gamma^{a}, \gamma^{b}\right] \tilde{\Omega}_{a b}+\gamma^{a} \tilde{\nabla}_{a} \psi+\psi^{\dagger} \gamma^{a} \tilde{\nabla}_{a}+\psi^{\dagger} \psi
$$

with the field strength $\tilde{\Omega}_{a b}=\left[\tilde{\nabla}_{a}, \tilde{\nabla}_{b}\right]$ including also the Riemann tensor on $M$ so that the second term in (37) contains the usual $R / 4$ contribution. Next we compare (37) with (34) and (35) to obtain

$$
\begin{aligned}
\omega_{a}=\tilde{\omega}_{a}-\frac{1}{2}\left(\gamma_{a} \psi+\psi^{\dagger} \gamma_{a}\right), & \\
E=-\frac{1}{4}\left[\gamma^{a}, \gamma^{b}\right] \tilde{\Omega}_{a b}-\psi^{\dagger} \psi+ & \frac{1}{2}\left(\left(\tilde{\nabla}^{a} \psi^{\dagger}\right) \gamma_{a}-\gamma_{a}\left(\tilde{\nabla}_{a} \psi\right)\right) \\
& -\frac{1}{4}\left(\gamma_{a} \psi+\psi^{\dagger} \gamma_{a}\right)^{2}-c .
\end{aligned}
$$

Our aim now is to obtain given $\omega$ and $E$ by adjusting $\psi$ and representation of the Clifford algebra. We are not able to present a closed form solution. We will rather argue that such a solution generically exists by using degrees of freedom counting arguments.

One can pass from one representation of the Clifford algebra to another by a local unitary transformation $\gamma^{a} \rightarrow U^{\dagger}(x) \gamma^{a} U(x), U^{\dagger}=U^{-1}$. By transforming also $\tilde{\omega}$ and $\psi$ we can force the operators $P$ and $P^{\dagger}$ to transform homogeneously: $P \rightarrow U^{\dagger} P U, P^{\dagger} P \rightarrow U^{\dagger} P^{\dagger} P U$. To analyse solutions on the equation (34) we may fix a representation of the Clifford algebra and use the corresponding freedom to gauge transform the operator $D: D \rightarrow U D U^{\dagger}$. Therefore, we can gauge 
fix $\omega_{1}$ to coincide with the right hand side of second of the two equations (38) containing yet unspecified function $\psi$. If $d$ is the target space dimension, the matrix $\psi$ contains $d^{2}$ independent components. Since the operator $D$ is hermitian, the connection $\omega_{a}$ is typically antihermitian and therefore contains $d(d-1) / 2$ independent components. The matrix valued field $E(x)$ should be hermitian, and thus it contains $d(d+1) / 2$ independent entries. Total number of the degrees of freedom needed to represent $\omega_{2}$ and $E$ is $d^{2}$ at each coordinate point. This is exactly the freedom contained in $\psi$. We conclude that it should be possible locally to represent an arbitrary Laplace type operator $D$ through the Dirac one, as in eq. (34).

Our method is not constructive. Actually it may be extremely hard to solve the equations (38). Fortunately, as we argue at the end of sec. 2, explicit solution may be not necessary.

\section{References}

[1] J. Dai, R. G. Leigh and J. Polchinski, New Connections Between String Theories, Mod. Phys. Lett. A4 (1989) 2073.

[2] R. G. Leigh, Dirac-Born-Infeld Action From Dirichlet Sigma Model, Mod. Phys. Lett. A4 (1989) 2767.

[3] J. Polchinski, Dirichlet-Branes and Ramond-Ramond Charges, Phys. Rev. Lett. 75 (1995) 4724 [hep-th/9510017.

[4] M. F. Atiyah, V. K. Patodi and I. M. Singer, Spectral Asymmetry And Riemannian Geometry 1, 2, 3 Math. Proc. Cambridge Phil. Soc. 77 (1975) 43; 78 (1976) 405; 79 (1980) 71.

[5] G. Grubb and R. T. Seeley, Weakly parametric pseudodifferential operators and Atiyah-Patodi-Singer boundary problem, Inv. Math. 121 (1995) 481.

[6] S. Dowker, P. Gilkey and K. Kirsten, Heat asymptotics with spectral boundary conditions, in Geometric Aspects of Partial Differential Equations, Contemporary Mathematics 242 (1999) AMS, 107-124 [hep-th/0004020].

[7] A. V. Mishchenko and Y. A. Sitenko, Spectral boundary conditions and index theorem for two-dimensional compact manifold with boundary, Annals Phys. 218 (1992) 199.

[8] H. Falomir, R. E. Gamboa Saravi and E. M. Santangelo, J. Math. Phys. 39 (1998) 532 [hep-th/9609194.

[9] G. Esposito, Dirac operator and spectral geometry, Cambridge Lecture Notes in Physics, v.12, Cambridge Univ. Press, 1998. 
[10] P.B. Gilkey, Invariance Theory, the Heat Equation, and the Atiyah-Singer Index Theorem, (CRC Press, Boca Raton, 1994).

[11] W. Kummer and D. V. Vassilevich, Renormalizability of the open string sigma model and emergence of D-branes, JHEP0007 (2000) 012 hepth/0006108.

[12] H. Osborn, Weyl consistency conditions and a local renormalization group equation for general renormalizable field theories, Nucl. Phys. B363 (1991) 486.

[13] V. Schomerus, D-branes and deformation quantization, JHEP9906 (1999) 030 [hep-th/9903205].

[14] N. Seiberg and E. Witten, String theory and noncommutative geometry, JHEP9909 (1999) 032 [hep-th/9908142.

[15] P. Horava, Background Duality Of Open String Models, Phys. Lett. B231 (1989) 251.

[16] M. B. Green, Space-time duality and Dirichlet string theory, Phys. Lett. B266 (1991) 325.

[17] E. Alvarez, J. L. Barbon and J. Borlaf, T-duality for open strings, Nucl. Phys. B479 (1996) 218 hep-th/9603089.

[18] H. Dorn and H. J. Otto, On T-duality for open strings in general abelian and nonabelian gauge field backgrounds, Phys. Lett. B381 (1996) 81 hepth/9603186.

[19] S. Förste, A. A. Kehagias and S. Schwager, Non-Abelian Duality for Open Strings, Nucl. Phys. B478 (1996) 141 hep-th/9604013.

[20] T. H. Buscher, A Symmetry Of The String Background Field Equations, Phys. Lett. B194 (1987) 59; Path Integral Derivation Of Quantum Duality In Nonlinear Sigma Models, Phys. Lett. B201 (1988) 466.

[21] A. S. Schwarz and A. A. Tseytlin, Dilaton shift under duality and torsion of elliptic complex, Nucl. Phys. B399 (1993) 691 [hep-th/9210015].

[22] D. V. Vassilevich and A. Zelnikov, Discrete symmetries of functional determinants, Nucl. Phys. B594 (2001) 501 hep-th/0009084.

[23] T. Branson and P. Gilkey, Residues of the eta function for an operator of Dirac type, J. Funct. Anal. 108 (1992) 47. 